\title{
Design of a microwave array hyperthermia applicator with a semicircular reflector
}

\begin{abstract}
R.V. Sabariego $\quad$ L. Landesa $\quad$ F. Obelleiro
Dpto. Tecnoloxías das Comunicacións, E.T.S.E. Telecomunicación, Universidade de Vigo, 36200-Vigo, Spain

Abstract-The design of a hyperthermia applicator for heating biological tissues is presented in which the applicator consists of an array antenna surrounded by a perfect electrically conducting reflector. The heat hazard to superficial tissues is reduced by the introduction of a dielectric protecting layer over them. A method of moments formulation is applied to approximate the electric field within the biological medium and a closed form expression is presented for the electromagnetic coupling problem, which enables an optimisation procedure to be performed. The applicator enhances both penetration and focusing: deep tumours, close to the bone region, are heated and the percentage of biologically healthy tissue exposed to a specific absorption rate (SAR) hazard level diminishes by $53.8 \%$.
\end{abstract}

Keywords-Hyperthermia, Array antenna, Method of moments, Conjugate field matching

Med. Biol. Eng. Comput., 1999, 37, 612-617

\section{Introduction}

CURRENTLY, THREE primary means of treating cancer exist: surgery, radiation and chemotherapy. The use of heat to treat cancer is a relatively new modality, still in its experimental stages, despite the fact that knowledge of its benefits is both ancient and extensive.

The most challenging but beneficial use of medical heating is hyperthermia cancer treatment. Hyperthermia can be defined as excessive heat in humans, above $41^{\circ} \mathrm{C}$, induced with therapeutic intent.

The clinical objective of hyperthermia treatment is to produce enough heat to kill cancer cells without damaging, significantly, normal benign cells. In general, malignant cells are more sensitive to heat in the range $41-45^{\circ} \mathrm{C}$ than are normal cells. In addition, most clinically apparent tumours have blood perfusion rates less than $20 \%$ that of surrounding normal tissue, meaning that they may be preferentially heated (JOINES et al., 1994). The minimum temperature for therapeutic benefit was established to be $41.8^{\circ} \mathrm{C}$ following studies undertaken since 1971 (RAPPAPORT, 1989). Above $46^{\circ} \mathrm{C}$ the time for cell killing becomes quite short (minutes) and the different sensitivity of malignant and benign cells disappears; above $50^{\circ} \mathrm{C}$ all cells are killed very quickly (GORDON SHORT and TURNER, 1980).

The choice of an accurate and proper method to induce heat is a critical factor in hyperthermia. Physical techniques for hyperthermia include metabolic heat, conduction through the

Correspondence should be addressed to Dr F. Obelleiro email: obi@tsc.uvigo.es

First received 19 April 1999 and in final form 18 May 1999

(C) IFMBE: 1999 skin, perfusion of externally heated blood, heated intravenous fluids and anaesthetic gases, ultrasound and electromagnetic (EM) coupling modalities (ADAIR, 1983). Related to the direct introduction of a device into the body, another classification exists: techniques may be invasive or non-invasive. EM modalities include capacitive, inductive (KOTSUKA et al., 1996), and UHF-microwave radiative techniques (REUTER et al., 1998).

Studies of EM field interactions with biological systems date back at least to the 1700s when Galvani and Volta, among others, experimented with electrical effects in frogs' legs, and Mesmer used magnets to treat patients (DURNEY, 1992). Since that time, electrical processes inherent to biological systems and various medical and biological applicators of EM fields have been studied extensively. In addition, the recent increase in mobile communications has resulted in a large number of preventive studies concerning the effects of EM radiation on the head (GANDHI et al., 1996; OKONOIEWSKI and STUCHLY, 1996).

Some researchers have tried to concentrate the EM power generated by an array antenna for hyperthermia applications (DURNEY, 1992; Lo et al., 1966; LOANE III and LEE, 1989; RAPPAPORT, 1989). In Lo et al. (1966) the energy is focused at a point within the near field region generalising the previous developments for the far field and one polarisation. It is also worth mentioning the work of RAPPAPORT (1989), in which a spherical and homogeneous tissue geometry is employed in an effort to determine an optimal source distribution, and also a power limit constraint is imposed on the superficial area.

Reflectors have been used to enhance the focusing of array antennas (HORNBACK, 1984; FENN et al., 1993, 1994; REUTER et al., 1994). In FENN et al. (1993), a $915 \mathrm{MHz}$ monopole phased array antenna design with a conducting reflector is considered for brain tumour heating. A similar device is 


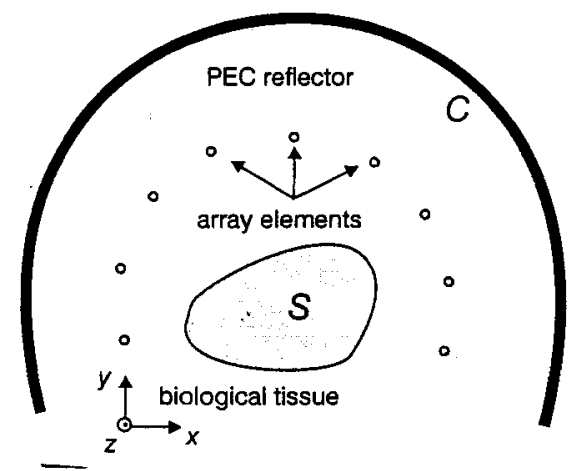

Fig. 1 Particular coupling problem

employed to optimise the specific absorption rate (SAR) in the focus region (FENN et al., 1994) by adjusting the phase of each monopole using a gradient search algorithm.

The present goal is to design a microwave applicator for hyperthermia which preserves healthy tissue while the EM energy attacks the tumour region. The proposed microwave hyperthermia applicator comprises a set of antennas (array antenna) and a perfect electrically conducting (PEC) reflector around the biological tissue, similar to that described in (FENN et al. (1993, 1994), as depicted in Fig. 1.

A practical model of this device would consist of a semicylindrical array antenna with $z$-polarisation (e.g. a set of dipoles) surrounded by a $z$-axis semicylindrical reflector. The pattern of each element of the array must be omnidirectional in the $x y$ plane and directive in the remaining planes ( $x z$ and $y z$ ). The main constraint is to select an antenna type that provides a directive pattern which avoids heating undesired tissue. For the sake of simplicity our analysis is restricted to a two-dimensional model invariant in the $z$-direction

Although the array antenna, with focusing enhanced by use of the PEC reflector, has demonstrated the capability of focusing an EM field in superficial biological tissue, an unwanted temperature rise occurs in the edge area when trying to heat deeper tumours. A dielectric protecting layer or bolus is included and optimised to avoid the excessive heating of these healthy superficial tissues. A method for synthesising the amplitude and phase of each element of the array antenna is presented.

\section{Coupling between the hyperthermia applicator and the biological tissue}

The EM coupling between the applicator and the tissue is modelled through the 'electric field integral equation' (EFIE) obtained from two $E$-field boundary conditions: the boundary condition on the PEC reflector and the boundary condition in the dielectric material (biological tissue). This equation is solved numerically using the Method of Moments (MoM) (HARRINGTON, 1993; RICHMOND, 1965).

Consider an $N$-element array antenna with a PEC around it, and biological tissue within its near field region (Fig. 1). It is assumed that there is no variation in the $z$-axis, and that the electric field has only a $z$-component, so the analysis is restricted to two-dimensional (2D) problems with transverse magnetic (TM) polarisation. Otherwise, an $e^{j \omega t}$-time variation is also assumed and suppressed in the rest of the paper (where $\omega$ is the angular frequency).

Let $E^{i}$ represent the incident field due to the array radiation in free space (without obstacles). The presence of obstacles, namely the PEC reflector and the dielectric biological tissue, produces associated scattered fields ( $E^{\text {s,pec }}$ and $E^{\text {s,die }}$ ) giving the total scattered field, $E^{s}$ :

$$
E^{s}=E^{s, p e c}+E^{s, d i e}
$$

Thus, the total electric field $E$ can be obtained as

$$
E=E^{i}+E^{s}
$$

The electric field on the PEC surface, $C$, induces surface currents, $J_{s}$, whose radiation at a given observation point, $\bar{\rho}$, is the scattered field due to the PEC, $E^{s, p e c}$, and can be written as

$$
E^{s, p e c}(\bar{\rho})=\frac{-k \eta_{0}}{4} \int_{C} J_{s}\left(\bar{\rho}^{\prime}\right) H_{0}^{(2)}\left(k\left|\bar{\rho}-\bar{\rho}^{\prime}\right|\right) \mathrm{d} c^{\prime}
$$

where $k=2 \pi \mid \lambda$ is the wavenumber ( $\lambda$ is the wavelength), $\eta_{0}=\mu_{0} / \epsilon_{0} \approx 120 \pi$ is the intrinsic impedance of free space and $H_{0}^{(2)}$ represents the Hankel function of the second kind, zero order.

The contribution of the biological tissue to the scattered field is obtained following an analogous procedure. In this case, the scattered field, $E^{s, d i e}$, can be generated by an equivalent electric current, $J$ (polarisation current), inside the biological tissue:

$$
E^{s, d i e}(\bar{\rho})=-\frac{k \eta_{0}}{4} \iint_{S} J\left(\bar{\rho}^{\prime} H_{0}^{(2)}\left(k \mid \bar{\rho}-\bar{\rho}^{\prime}\right) \mathrm{d} s^{\prime}\right.
$$

This polarisation current is related to the total electric field inside the dielectric (RICHMOND, 1965) by the following expression:

$$
J=j \omega \epsilon_{0}\left(\epsilon_{r}-1\right) E^{t o t}
$$

where $\omega$ is the angular frequency $2 \pi f$, and $\epsilon_{r}$ is the relative complex permittivity characteristic of the different biological materials:

$$
\epsilon_{r}\left(\rho^{\prime}\right)=\epsilon_{r}^{\prime}\left(\bar{\rho}^{\prime}\right)-j \frac{\omega}{\sigma}
$$

Thus, the electric field boundary condition on the PEC reflector surface can be expressed as follows:

$$
E^{\text {tot }}=E^{i}+E^{s . p e c}+E^{\text {s.die }}=0 \text { on } C
$$

that is, the tangential electric field vanishes on $C$.

Therefore, the remainder of the EFIE is obtained by imposing the analogous condition

$$
E^{\text {tot }}=E^{i}+E^{s, p e c}+E^{s, d i e} \text { in } S
$$

in the biological tissue.

Eqns. 7 and 8 constitute two integral equations that must be solved for the unknowns $J$ and $J_{s}$ (or $E^{\text {tot }}$ ) by the well-known MoM. The simplest procedure is to discretise the contour $C$ and the surface $S$ in a set of segments and cells, respectively, and assume the currents and fields to be constant over these domains (see Fig. 1). This is equivalent to expanding the unknowns $J$ and $J_{s}$ (or $E^{\text {tot }}$ ) in terms of pulse functions. Finally, the resultant discrete equations are imposed at a set of matching points on $C$ and within $S$, selected at the centre of the previously defined domains (see Fig. 2). This leads to the following matrix equations that must be solved instead of eqns. 7 and 8:

$$
\begin{aligned}
& {\left[Z_{P E C}\right] j_{P E C}=-\left[Z_{P E C}^{a}\right] a-\left[Z_{P E C}^{d}\right] e_{d}} \\
& {\left[Z_{d}-I\right] e_{d}=-\left[Z_{d}^{a}\right] a-\left[Z_{d}^{P E C}\right] j_{P E C}}
\end{aligned}
$$

where $a$ is a column vector containing the excitations of the array elements, $j_{P E C}$ is a column vector which represents the electric currents $J_{s}$ at the matching points on the PEC surface 


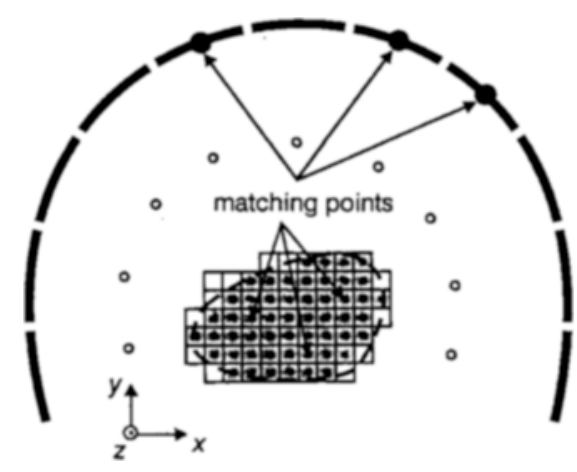

Fig. 2 Discretised coupling problem

$C$, and $e_{d}$ is a column vector containing the total field $E^{\text {tot }}$ in the matching points inside the tissue. The remaining terms of the equation are impedance matrices: $\left[Z_{P E C}\right]$ and $\left[Z_{d}\right]$ represent the self-influence of the PEC surface and the dielectric area, respectively. The other matrices show the mutual influence between the components (superscript over subscript) of the whole EM problem under analysis; e.g. $\left[Z_{P E C}^{a}\right]$ shows the effect of the array on the PEC reflector. To conclude, matrix $I$ is the identity matrix.

From eqns. 9 and 10 and after some algebra, the total field within the tissue can be obtained directly as a function of the array coefficients as follows:

$$
\begin{aligned}
e_{d}= & \left\{\left[Z_{d}-I\right]-\left[Z_{d}^{P E C}\right]\left[Z_{P E C}\right]^{-1}\left[Z_{P E C}^{d}\right]\right\}^{-1}\left\{\left[-Z_{d}^{a}\right]\right. \\
& \left.+\left[Z_{d}^{P E C}\right]\left[Z_{P E C}\right]^{-1}\left[Z_{P E C}^{a}\right] z\right\} a
\end{aligned}
$$

\section{Optimisation process}

The methods applied to determine the optimum excitations of the array are 'conjugate field matching' (CFM) and 'near field power maximisation' (NFPM). CFM and 'gain optimisation' (similar to NFPM) were used in LOANE and LEE (1989) to focus the near electric field inside a dielectric material. Both techniques obtain a directive pattern from radiation at the focus of each array element. CFM assigns to each element the conjugate of the field radiated by this element at the focus, but this procedure does not consider power coupling among elements. The proposed NFPM method solves this problem by introducing a power coupling matrix.

The near field radiated by each array element must be calculated taking into account the presence of the biological tissue. The excitation coefficient of each element will be the conjugate of this value. The combination of the near field reciprocity CFM approach and eqn. 11 leads to the following expression for the array excitations:

$$
\begin{aligned}
a_{C F M}= & \left(l [ [ Z _ { d } = I ] - [ Z _ { d } ^ { P E C } ] [ Z _ { P E C } ] ^ { - 1 } [ Z _ { P E C } ^ { d } ] \} ^ { - 1 } \left\{\left[-Z_{d}^{a}\right]\right.\right. \\
& \left.\left.+\left[Z_{d}^{P E C}\right]\left[Z_{P E C}\right]^{-1}\left[Z_{P E C}^{a}\right]\right\}\right)^{H}
\end{aligned}
$$

where $H$ represents the transpose conjugate operator and $l$ is a vector containing the points of interest inside the tissue, i.e. the tumour it is desired to destroy.

$$
l_{i}= \begin{cases}1 & \text { matching-point in malignant tissue } \\ 0 & \text { matching-point in healthy tissue }\end{cases}
$$

The CFM solution given by eqn. 12 does not consider the coupling among array element patterns, so there is no power optimisation. The optimum solution requires a better approach incorporating this coupling. Eqn. 12 is modified by a square matrix, $G$, that contains the coupling between the primary patterns of each array element. This coupling matrix does not depend on array excitations; it is already known for a particular geometry and pattern element. The matrix components are

$$
g_{i j}=\frac{1}{2 \eta_{0}} \int_{0}^{2 \pi} f_{i}^{*}\left(\phi f_{j}(\phi) \mathrm{d} \phi\right.
$$

where $f_{i}(\phi)$ is the $i$-element far field radiation.

The final solution achieved by this method is

$$
\begin{aligned}
a_{N F P M}= & \left(G^{H}\right)^{-1}\left(l\left\{\left[Z_{d}-I\right]-\left[Z_{d}^{P E C}\right]\left[Z_{P E C}\right]^{-1}\left[Z_{P E C}^{d}\right]\right\}^{-1}\right. \\
& \left.\left\{\left[-Z_{d}^{a}\right]+\left[Z_{d}^{P E C}\right]\left[Z_{P E C}\right]^{-1}\left[Z_{P E C}^{a}\right]\right\}\right)^{H}
\end{aligned}
$$

A relationship between eqns. 12 and 15 can be observed:

$$
a_{N F P M}=\left(G^{H}\right)^{-1} a_{C F M}
$$

\section{Numerical results}

The capabilities of the applicator designed are illustrated for a two-dimensional example. The tissue under test is a set of four layers with elliptical concentric geometry simulating a transversal cut of a limb. The geometry and its dimensions are shown in Fig. 3.

The 'specific absorption rate' (SAR) is the measure selected to present the results since it measures the power absorbed by the tissue, which is closely related to its increase in temperature. It depends on the $E$-field and the particular bio-electrical characteristics of the tissue (conductivity $\sigma$ and density $\rho$ ).

$$
S A R=\frac{\sigma}{\rho}\left|E^{t o t}\right|^{2}
$$

The behaviour of biological tissue varies widely with frequency. Given the high water content, conductivity rises as frequency rises, so penetration decreases (because of increased losses). In contrast, focusing improves. At lower frequencies, however, it is possible to reach deeper tumours although focusing becomes worse. Frequencies most commonly used in medicine applications vary between 100 and $1000 \mathrm{MHz}$ (RAPPAPORT, 1989). The value $915 \mathrm{MHz}$ was chosen as the working frequency because it provides penetration and focusing compatible with medical constraints. In addition, its extensive use in mobile communications has

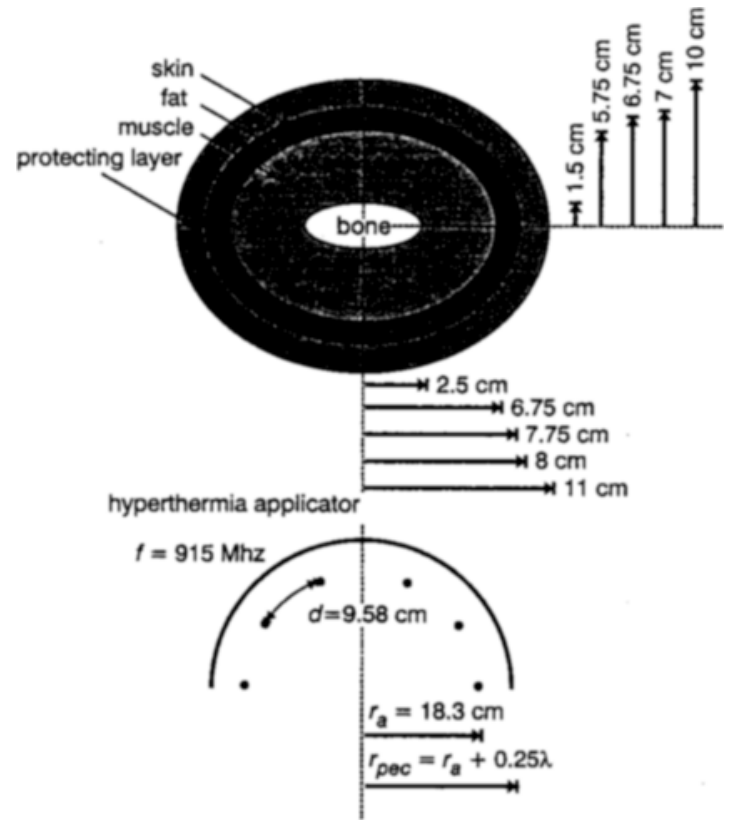

Fig. 3 Geometric dimensions 
Table 1 Properties of biological tissues at $915 \mathrm{MHZ}$.

\begin{tabular}{lcrc}
\hline Tissue & $\begin{array}{c}\text { Density } \\
\left(10^{3} \mathrm{~kg} \mathrm{~m}^{-\hat{3}}\right)\end{array}$ & $\begin{array}{c}\text { Permitivity } \\
\epsilon_{r}^{\prime}\end{array}$ & $\begin{array}{c}\text { Conductivity } \\
\sigma(\mathrm{S} / \mathrm{m})\end{array}$ \\
\hline Muscle & 1.04 & 58 & 1.4 \\
Fat & 0.92 & 6 & 0.08 \\
Bone & 1.85 & 8 & 0.11 \\
Skin & 1.10 & 35 & 0.6 \\
\hline
\end{tabular}

resulted in a number of studies that required a knowledge of the electrical characteristics of biological tissue (Table 1).

In all the examples presented, the dynamic range of SAR is $60 \mathrm{~dB}$, the lowest values corresponding to low temperatures (dark blue) and the highest values to high temperatures (dark red). The $50 \%$ SAR level corresponds to $-3 \mathrm{~dB}$ and the $25 \%$ SAR level corresponds to $-6 \mathrm{~dB}$. The $25 \%$ iso-SAR is represented by a contour line in Figs. 5-8.

Fig. 4 shows the particular configurations employed in the computer simulations. In the first approach, the biological dielectric was irradiated by a circular array with $\mathrm{PEC}$ around. It must be pointed out that an open circular reflector was chosen to avoid power concentration and to ease array feeding (Fig. $4 a$ and $b$ ). In the second approach the initial design was substituted by a semicircular model, which provides a clear enhancement (Fig. $4 c$ and $d$ ). The applicator position depends directly on the tumour position.

Fig. 5 shows the SAR within the tissue corresponding to the configuration depicted in Fig. $4 a$. In this case, the focus region is placed in a deep muscle area at the edge of bone. This focus is depicted by a grid of dots which represents the matching points in the tumour. Although power is delivered to the tumour area, unwanted heating of superficial tissues is observed.

To avoid this harmfil effect the introduction of a biologically compatible bolus (non-toxic) has been implemented; the biomedical material chosen is a gel characterised by $\epsilon_{r^{\prime}}^{\prime}=4$, $\sigma=0.1 \mathrm{~S} \mathrm{~m}^{-1}$ and $\rho=2070 \mathrm{~kg} \mathrm{~m}^{-3}$. Although this gel dissipates the heating, a ventilation system would improve the proposed applicator by providing a more controlled tempera-
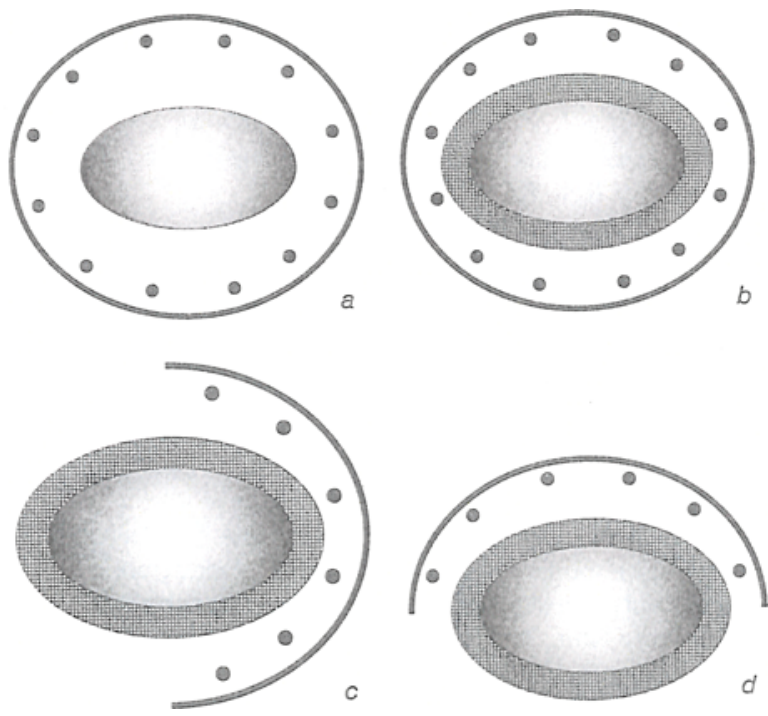

Fig. 4 Configuration: relative position applicator-tissue. (a) Circular array and PEC. Number of elements: 12. No protecting layer. (b) Circular array and PEC. Number of elements: 12. Protecting layer: $3 \mathrm{~cm}$. (c) Semicircular array and $P E C$. Number of elements: 6. Protecting layer: $3 \mathrm{~cm}$. (d) Semicircular array and PEC. Number of elements: 6. Protecting layer: $3 \mathrm{~cm}$

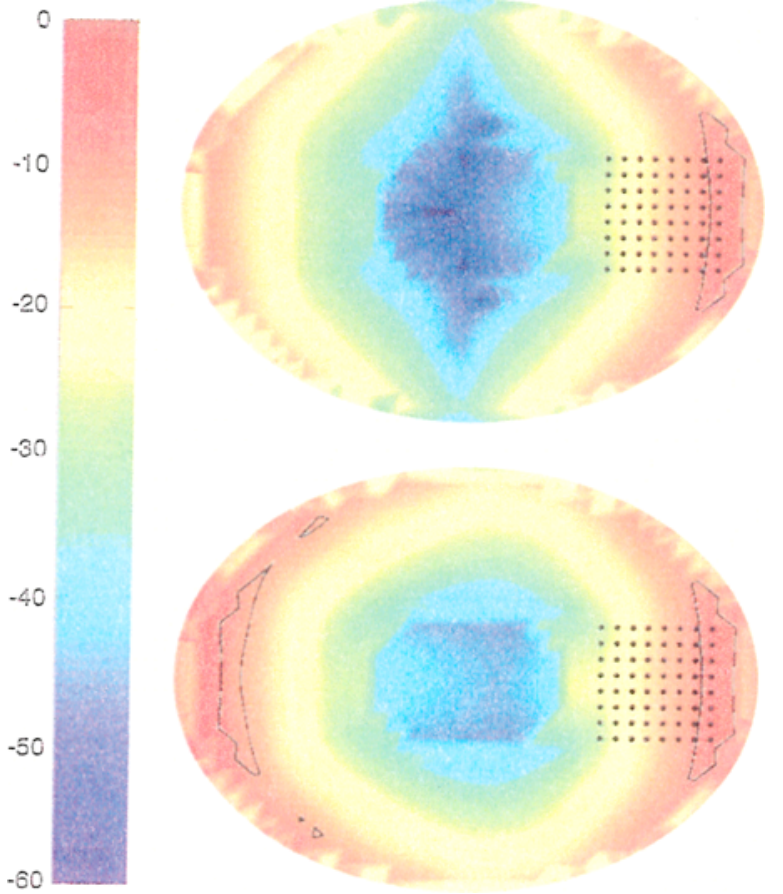

Fig. 5 SAR within biological tissue. CFM method. Configuration as in Fig. 4 a. Contour line represents the 25\% iso-SAR

ture. Fig. 6 illustrates the SAR obtained with the configuration shown in Fig. $4 b$; it can be seen that the excess heating in unwanted tissue is reduced.

Quantitatively, the improvement achieved by the introduction of the dielectric protecting layer can be expressed by comparing the percentage of biological tissue exposed to a particular level of SAR with and without bolus. For instance,

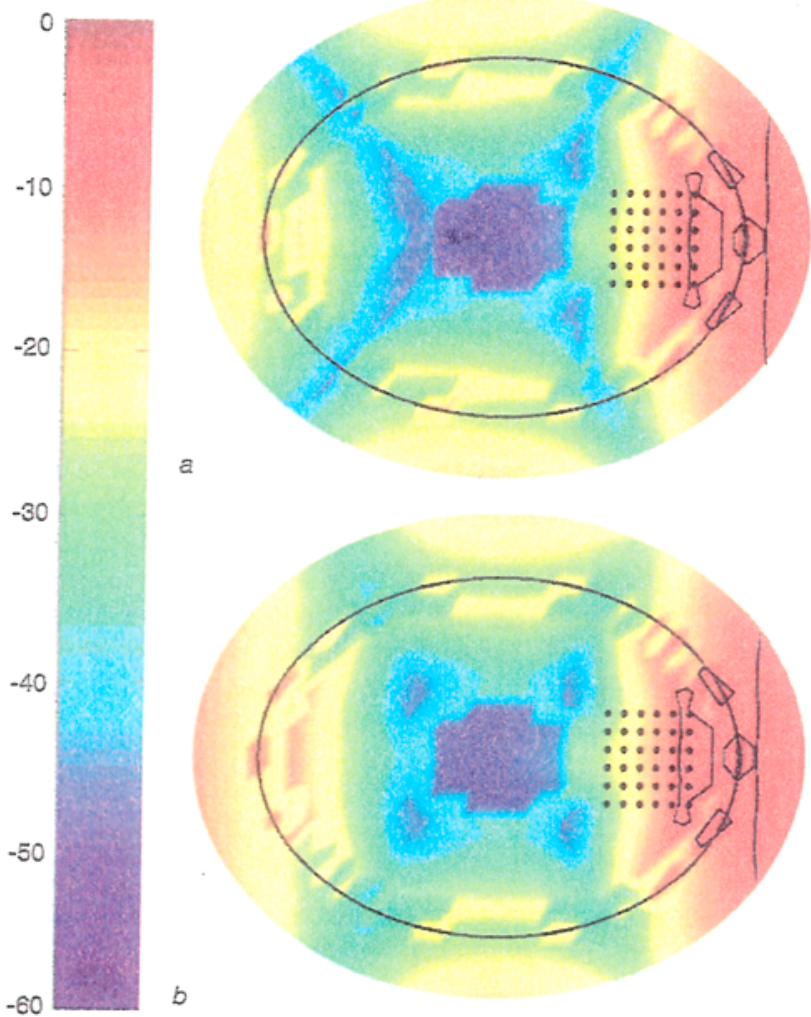

Fig. 6 SAR within biological tissue and dielectric protecting layer. Configuration as in Fig. 4b. (a) CFM method; (b) NFPM method. Contour line represents the $25 \%$ iso-SAR. 


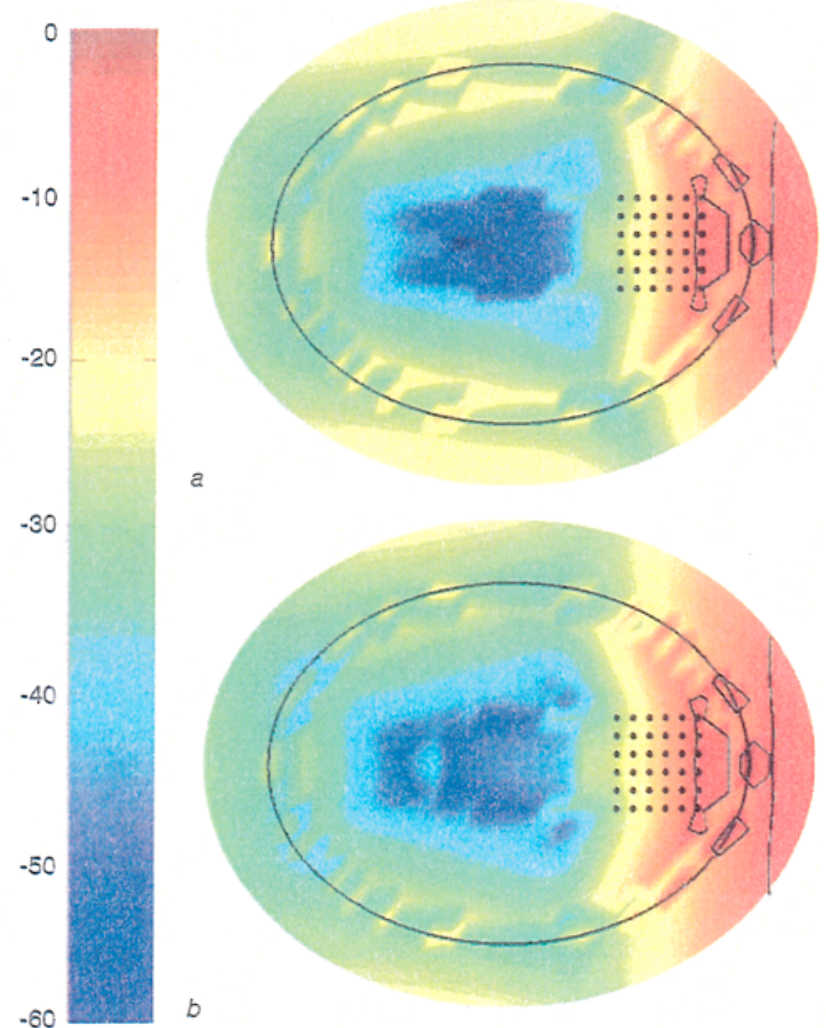

Fig. 7 SAR within biological tissue and dielectric protecting layer. Configuration as in Fig. 4c. (a) CFM method (b) NFPM method. Contour line represents the 25\% iso-SAR.

the percentage of biological tissue with SAR level higher than $-15 \mathrm{~dB}$ diminished from $20.2 \%$ (Fig. 5, without bolus) to $10.9 \%$ (Fig. 6, with bolus) when dealing with CFM (a $46.2 \%$ reduction in the affected area) and, analogously, from $31.9 \%$ to $11.7 \%$ in the NFPM case (a reduction of $63.2 \%$ ). Nevertheless, a high level of SAR (maximum of $-18.5 \mathrm{~dB}$ and $-15 \mathrm{~dB}$ for CFM and NFPM, respectively) can be observed in the zone just opposite the tumour, which is still a dangerous hazard. It was necessary to overcome this drawback, so a semicircular geometry was implemented (Fig. $4 c$ and $d$ ). Fig. 7 shows the results obtained with the semicircular applicator of Fig. $4 c$. In this case, the levels of SAR in remote areas are acceptable from a medical point of view, i.e. the SAR is maintained at a safe level, being reduced from the maximum level in this region by up to $-25 \mathrm{~dB}$ (CFM case) and $-27.4 \mathrm{~dB}$ (NFPM case). This example also illustrates the improvement given by the proposed NFPM method with respect to the CFM solution, i.e. NFPM achieves a $12.4 \mathrm{~dB}$ reduction in power while CFM gives a $6.5 \mathrm{~dB}$ decrease.

It is worth mentioning that it is much easier to obtain a selective increment in temperature when we are concerned with superficial tumours. This effect can be observed in Fig. 8, where a semicircular hyperthermia applicator has been used (Fig. $4 d$ ).

\section{Conclusions}

A hyperthermia applicator for heating biological tissue has been designed. The synthesis developed combines the MoM with an optimisation technique, and is presented in a closed formulation. Special attention is paid to such important aspects as depth of penetration and power limitation within and on the biological tissue surface because of medical constraints in temperature rise. Numerical results have shown the capabilities

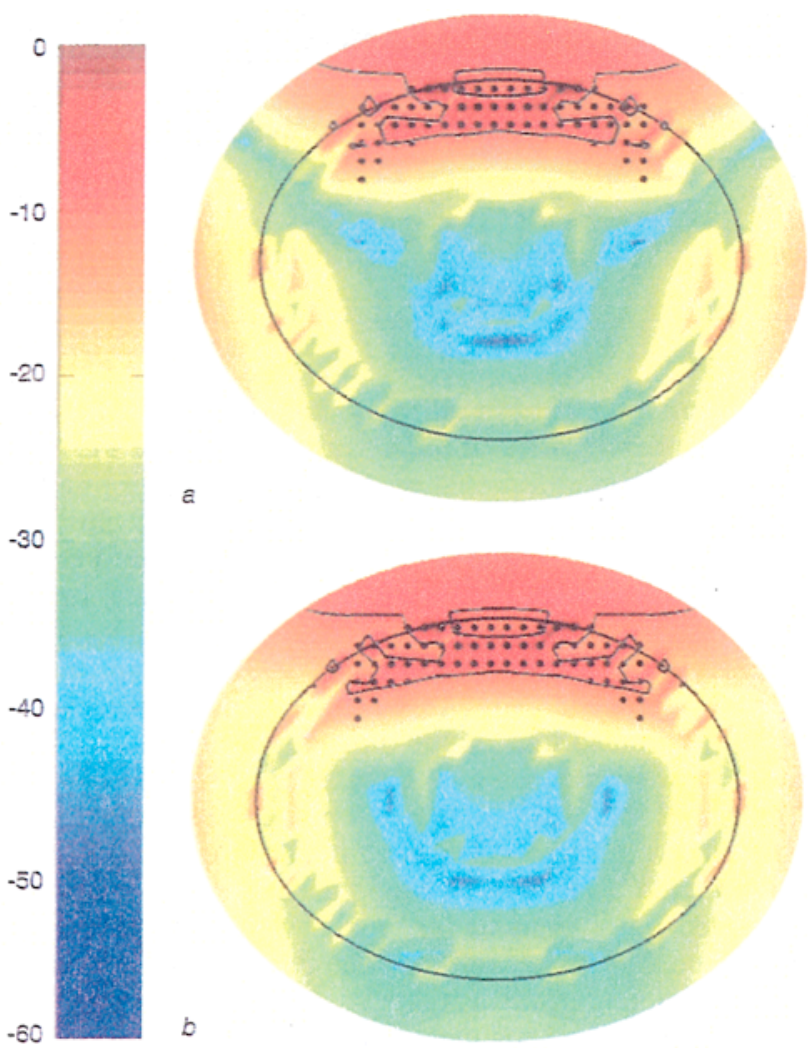

Fig. 8 SAR within biological tissue and dielectric protecting layen Configuration as in Fig. 4d. (a) CFM method (b) NFPM method. Contour line represents the $25 \%$ iso-SAR.

of the proposed design in terms of enhanced penetration. Although the proposed applicator has been shown to provide good results for superficial and intermediate regions, up to now it has not been possible to reach tumours in deeper regions (the bone region in our model) because of excessive power losses in shallower tissue layers.

This method could be extended to complex geometries including other kinds of tissue and adapted to three-dimensional problems. In summary, electromagnetic hyperthermia offers a promising modality for treating cancer in humans, with much research still to be done.

Acknowledgment-This work has been supported by the Spanish CICYT, Project Ref. TIC97-0821-CO2-01.

\section{References}

ADAIR, E. R. (1983): 'Microwaves and thermoregulation' (Academic Press), pp. 57-93

DURNEY, C. H. (1992): 'Antennas and other electromagnetic applicators in biology and medicine', Proc. IEEE, 80, pp. 194 199

FenN, A. J., Poe, D. S., Reuter, C. E., and TAflove A. (1993) 'Noninvasive monopole phased array for hyperthemia treatment of cranial-cavity and skull-based tumors: design, analysis and phantom tests', Proc. Ann. Int. Conf. IEEE Engineering in Medicine and Biology Society, 15, pp. 1453-1454

FENN, A. J., CHEUNG, A. Y., and CAO H. (1994): 'Adaptive focusing experiments for minimally invasive monopole phased arrays in hyperthermia treatment of breast cancer', Proc. Ann. Int. Conf. IEEE Engineering in Medicine and Biology Society, 16, pp. 766767

GANDHI, O. P., LAZZI, G., and FURSE C. M. (1996): 'Electromagnetic absorption in the human head and neck for mobile telephones at 835 and $1900 \mathrm{MHz}$, IEEE Trans. Microw. Theory Tech., 44, pp. 1884 1897 
GORDON SHORT, J., and TURNER P. F. (1980): 'Physical hyperthermia and cancer therapy', Proc. IEEE, 68, pp. 133-142

Harrington, R. F.(1993): 'Field computation by moment methods' (IEEE Press, New York)

HORNBACK, N. B. (1984): 'Hyperthermia and cancer: human clinical trial experience', Vol. 2 (CRC Press, Boca Raton)

JOINES, W. T., YANG ZHANG, CHENXING LI, and JIRTLE, R. L. (1994): 'The measured electrical properties of normal and malignant human tissues from 50 to $900 \mathrm{MHz}$ ', Med. Phys., 21, pp. 547-550

KotSUKA, Y., HANKUI, E., and SHIGEMATSU, Y. (1996): 'Development of ferrite core applicator system for deep-induction hyperthermia', IEEE Trans. Microw. Theory Tech, 44, pp. 1803-1809

LO, Y. T., LEE, S-W, and LEE Q. H. (1966): 'Optimization of directivity and signal-to-noise ratio of an arbitrary antenna array', Proc. IEEE, 54, pp. 1033-1045

LOANE III, J. T., and SHUNG-WU LEE (1989): 'Gain optimization of a near-field focusing array for hyperthermia applications', IEEE Trans. Microw. Theory Tech. 37, pp. 1629-1635

OKONIEWSKI, M., and STUCHLY M. A. (1996): 'A study of the handset antenna and human body interaction', IEEE Trans. Microw. Theory Tech., 44, pp. 1855-1864

RAPPAPORT C. M. (1989): 'Synthesis of optimum microwave antenna applicators for use in treating deep localized tumors', Chapter 2 in KONG, J. A. (Eds), 'Progress in-electromagnetics research' PIER $l$ (Elsevier, Cambridge)

Reuter, C. E., Thiele, E. T., TAflove, A., Piket-May, M., and FenN A. J. (1994): 'Linear superposition of phased array antenna near field patterns using the FD-TD method'. 10th Annual Review of Progress in Applied Computational Electromagnetics, Conference Proceedings, pp. 459-466

Reuter, C. E., Taflove, A., Sathiaseelan, V., Piket-May, M., and MITTAL B. B. (1998): 'Unexpected physical phenomena indicated by FDTD modeling of the Sigma-60 deep hyperthermia applicator', IEEE Trans. Microw. Theory Tech., 46, pp. 313-319

RICHMOND J. H. (1965): 'Scattering by a dielectric cylinder of arbitrary cross section shape', IEEE Trans. Antennas Propag., AP-13, pp. 334-341

\section{Author's biography}

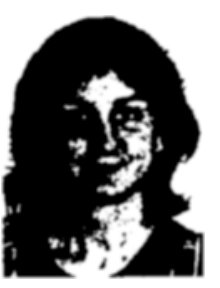

RUTH V. SABARIEGO was born in Vigo, Spain, in April 1974. She received her MSc in Electrical Engineering from the University of Vigo, Spain, in 1998 and is currently working towards her $\mathrm{PhD}$ degree with the Department of Tecnoloxias das Comunicacións at the University of Vigo. Her research interests include biomedical applications of EM energy, EM radiation hazards, array antennas, applied mathematics and numerical techniques for computational electromagnetics. 